\title{
A numerical investigation of the influence of windscreens on measurement of sound intensity
}

Juhl, Peter; Jacobsen, Finn

Published in:

Acoustical Society of America. Journal

Link to article, DOI:

10.1121/1.2151811

Publication date:

2006

Document Version

Publisher's PDF, also known as Version of record

Link back to DTU Orbit

Citation (APA):

Juhl, P., \& Jacobsen, F. (2006). A numerical investigation of the influence of windscreens on measurement of sound intensity. Acoustical Society of America. Journal, 119(2), 937-942. https://doi.org/10.1121/1.2151811

\section{General rights}

Copyright and moral rights for the publications made accessible in the public portal are retained by the authors and/or other copyright owners and it is a condition of accessing publications that users recognise and abide by the legal requirements associated with these rights.

- Users may download and print one copy of any publication from the public portal for the purpose of private study or research.

- You may not further distribute the material or use it for any profit-making activity or commercial gain

- You may freely distribute the URL identifying the publication in the public portal

If you believe that this document breaches copyright please contact us providing details, and we will remove access to the work immediately and investigate your claim. 


\title{
A numerical investigation of the influence of windscreens on measurement of sound intensity ${ }^{\text {a) }}$
}

\author{
Peter Juhl ${ }^{\text {b) }}$ \\ Physics Department, University of Southern Denmark, Campusvej 55, DK-5230 Odense M, Denmark
}

Finn Jacobsen ${ }^{\text {) }}$

Acoustic Technology, Ørsted $\bullet D T U$, Technical University of Denmark, Building 352, Ørsteds Plads, DK-2800

Kgs. Lyngby, Denmark

(Received 9 February 2005; revised 12 September 2005; accepted 23 November 2005)

\begin{abstract}
Sound intensity probes are often used with windscreens to minimize the effect of noise caused by airflow. A theoretical and experimental study of the effect of windscreens on $p-p$ intensity probes published ten years ago concluded that windscreens give rise to underestimation of the sound intensity at low frequencies in strongly reactive sound fields. The theoretical part of this study was based on the assumption of a windscreen of infinite extent. In this paper windscreens of realistic size and shape are dealt with by means of a coupled boundary element model for the windscreen and the surrounding air. The error of the estimated intensity caused by the windscreen is calculated under a number of sound field conditions of varying reactivity. It is shown that the resulting error can be much larger than the intensity itself in a very reactive sound field. It is also shown that the shape and size of the windscreen has a significant influence on the error. (C) 2006 Acoustical Society of America. [DOI: 10.1121/1.2151811]
\end{abstract}

PACS number(s): 43.58.Fm, 43.50.Yw [DKW]

Pages: 937-942

\section{INTRODUCTION}

Windscreens of porous foam are often placed around sound intensity probes to reduce their exposure to airflow and mechanical or thermal damage. ${ }^{1-3}$ However, windscreens have an undesired effect; a theoretical and experimental study of the effect of windscreens on sound intensity measurements with the two-microphone technique has shown that the use of a windscreen results in underestimation of the sound intensity at low frequencies in strongly reactive sound fields. ${ }^{4}$ The reason is that the losses of the foam gives rise to a phase error between the pressure and the particle velocity. ${ }^{4}$ The theoretical part of this study was based on the somewhat oversimplified assumption of a windscreen of infinite extent, and some of the experimental observations indicated a significant influence of the shape of the windscreen that evidently cannot be predicted by a theory that assumes that it is infinite. Thus the purpose of this paper is to examine the influence of windscreens of realistic size and shape on intensity measurements numerically.

\section{THEORY}

\section{A. Two coupled domains}

Consider a windscreen placed in an arbitrary sound field. This may be regarded as a problem that involves two coupled domains, the exterior sound field and the field inside the windscreen. In the domain exterior to the windscreen the

\footnotetext{
Portions of this work were presented in "A numerical investigation of the influence of windscreens on sound intensity measurements," Proceedings of 18th International Congress on Acoustics, Kyoto, Japan, April 2004.

${ }^{b)}$ Electronic mail: pmjuhl@fysik.sdu.dk

${ }^{c)}$ Electronic mail: fja@oersted.dtu.dk
}

air is assumed to be an ideal fluid. If the sound field is harmonic the velocity potential satisfies the Helmholtz equation

$$
\nabla^{2} \Psi_{e}+k_{e}^{2} \Psi_{e}=0,
$$

where $k_{e}$ is the wave number, which is real valued in this case. The sound pressure and the particle velocity are

$$
p_{e}=j \omega \rho_{e} \Psi_{e},
$$

and

$$
\mathbf{u}_{e}=-\nabla \Psi_{e},
$$

respectively, where $\rho_{e}$ is the density of air. Note that the $e^{j \omega t}$ convention is used in this paper.

The windscreen is assumed to be a porous medium as described by the simple phenomenological model of Zwikker and Kosten ${ }^{5}$ and Morse and Ingard. ${ }^{6}$ Such a medium is characterized by its porosity $\Omega_{i}$, compressibility $\kappa_{i}$, density $\rho_{i}$, and flow resistivity $\Phi_{i}$. In the following it is assumed that $\Omega_{i}=1$, that the density equal the corresponding value of ideal air, and that the compressibility equals the isothermal compressibility of air, so that the speed of sound

$$
c_{i}=1 /\left(\Omega_{i} \rho_{i} \kappa_{i}\right)^{1 / 2},
$$

is about $15 \%$ less than the speed of sound in the exterior domain $c_{e}$. It should be mentioned that many other, more accurate models of the acoustic properties of porous materials are available; see, e.g., Refs. 7 and 8. However, windscreens are made of highly porous foam with a low flow resistivity; their undesired effect on sound intensity measurement is negligible except at low frequencies; ${ }^{4}$ and it is known that the Zwikker and Kosten model is a "phenomenologically correct low-frequency approximation.", 
In what follows indices of quantities that have the same values in the two domains have been omitted. The single remaining parameter in the model of the windscreen is the flow resistivity $\Phi_{i}$, which enters into the equation of motion as follows: ${ }^{6}$

$$
j \omega \rho \mathbf{u}_{i}+\Phi_{i} \mathbf{u}_{i}+\nabla p_{i}=0 .
$$

The wave equation takes the form ${ }^{6}$

$$
\nabla^{2} \Psi_{i}+\left(\omega / c_{i}\right)^{2} \Psi_{i}-\frac{j \omega \Phi_{i}}{\rho c_{i}^{2}} \Psi_{i}=0
$$

and this can be transformed into the Helmholtz equation

$$
\nabla^{2} \Psi_{i}+k_{i}^{2} \Psi_{i}=0
$$

by defining a complex wave-number $k_{i}$ as

$$
k_{i}=\frac{\omega}{c_{i}} \sqrt{1-\frac{j \Phi_{i}}{\omega \rho}} .
$$

\section{B. Coupled boundary element model}

Since the exterior as well as the interior problem may be transformed into the regular Helmholtz equation, a standard Boundary Element Method (BEM) is employed for the numerical solution of the coupled problem. ${ }^{9}$ The wave number of the interior problem is complex, but since its imaginary part is relatively small no special care is needed in this respect. (A large imaginary part would require special care in the numerical integration, due to the rapid decrease of the exponential.) The direct collocation method is used with three-dimensional quadrilateral, isoparametric linear elements. Two different windscreens have been modeled, the Brüel and Kjær (B\&K) windscreen UA 0781, which is shaped like a prolate ellipsoid, and B\&K's spherical windscreen UA 0782. The ellipsoidal windscreen is $18.5 \mathrm{~cm}$ long and has a width of $9.3 \mathrm{~cm}$, and the spherical windscreen has a diameter of about $9.3 \mathrm{~cm}$. Both models consist of 384 elements and 386 nodes, since the mesh for the ellipsoidal windscreen is produced by a simple transformation of the spherical mesh. The maximum distance between two nodes is $18 \mathrm{~mm}$ for the ellipsoidal windscreen, which corresponds to about 19 nodes per wavelength in air at the highest frequency considered in this study, $1000 \mathrm{~Hz}$. In view of the normal rule of thumb of six nodes per wavelength and the fact that the effects studied in this work are most significant at frequencies below $100 \mathrm{~Hz}$, it is concluded that the numerical mesh is more than adequate. It is also worth mentioning that the problem of characteristic frequencies ${ }^{10}$ does not occur in coupled problems (and for an uncoupled problem the problem would occur at much higher frequencies, due to the size of the windscreens).

Once the exterior problem is discretized it becomes a matrix equation,

$$
\mathbf{C}_{e} \boldsymbol{\Psi}_{e}=\mathbf{A}_{e} \boldsymbol{\Psi}_{e}+\mathbf{B}_{e} \mathbf{u}_{e}+4 \pi \boldsymbol{\Psi}_{e}^{\mathrm{I}},
$$

which can be converted to a matrix equation for the sound pressure $\mathbf{p}_{e}$ by multiplying with $j \omega \rho$,

$$
\mathbf{C}_{e} \mathbf{p}_{e}=\mathbf{A}_{e} \mathbf{p}_{e}+j \omega \rho \mathbf{B}_{e} \mathbf{u}_{e}+4 \pi \mathbf{p}_{e}^{\mathrm{I}} .
$$

In Eq. (10) $\mathbf{C}_{e}$ is a diagonal matrix containing the solid angle occupied by the calculation domain at the nodes (i.e., $2 \pi$ for a smooth boundary), the matrix $\mathbf{A}_{e}$ contains integrals over the windscreen surface $S$ involving the normal derivative of the Green's function, and the matrix $\mathbf{B}_{e}$ contains surface integrals involving the Green's function. The incoming sound field at position $P$ is $\mathbf{p}_{e}^{\mathrm{I}}$ in the absence of the windscreen.

The interior problem can be converted to a matrix equation similar to Eq. (9), which is translated into the sound pressure $\mathbf{p}_{i}$ by multiplying with $j \omega \rho+\Phi_{i}$,

$$
\mathbf{C}_{i} \mathbf{p}_{i}=\mathbf{A}_{i} \mathbf{p}_{i}+\left(j \omega \rho+\Phi_{i}\right) \mathbf{B}_{i} \mathbf{u}_{i} .
$$

For both equations, Eqs. (10) and (11), the normal vector is defined so it points into the computational domain. Hence, the two domains share the surface $S$ in the Helmholtz integral equation, but with opposite normal vectors. The diagonal matrix $\mathbf{C}_{i}$ contains the solid angles at all nodes measured from the interior domain; hence, this matrix complements the $\mathbf{C}_{e}$ matrix so that the two matrices add up to a diagonal matrix with $4 \pi$ in all diagonal elements.

Continuity of the sound pressure and the particle velocity on the boundary of the windscreen leads to two equations

$$
\mathbf{p}_{e}=\mathbf{p}_{i}=\mathbf{p}
$$

and

$$
\mathbf{u}_{e}=-\mathbf{u}_{i},
$$

where the sign in the latter case is due to the change in normal direction on $S$ (when considering the exterior and interior problem respectively). Rearranging Eq. (11) gives

$$
\mathbf{u}_{i}=\frac{-1}{\left(j \omega \rho+\Phi_{i}\right)} \mathbf{B}_{i}^{-1}\left(\mathbf{A}_{i}-\mathbf{C}_{i}\right) \mathbf{p}
$$

and entering this result into Eq. (10) leads to a matrix equation for the pressure on the surface of the windscreen

$$
\left(\mathbf{A}_{e}-\mathbf{C}_{e}+\frac{j \omega \rho}{j \omega \rho+\Phi_{i}} \mathbf{B}_{e} \mathbf{B}_{i}^{-1}\left(\mathbf{A}_{i}-\mathbf{C}_{i}\right)\right) \mathbf{p}=-4 \pi \mathbf{p}_{e}^{\mathrm{I}} .
$$

To summarize, Eq. (15) is the coupled equation for the sound pressure on the surface of the windscreen involving both the interior domain (the windscreen) and the exterior domain (the surrounding air). The matrices involving the exterior problem (denoted by index $e$ ) are standard BEM matrices with a real wave number $k_{e}$, whereas the BEM equations for the interior problem (denoted by index $i$ ) take the losses due to the flow resistivity into account by means of a complex wave number $k_{i}$.

Two limiting cases of Eq. (15) are of interest. If the flow resistivity $\Phi_{i}$ tends toward infinity the windscreen becomes in effect impenetrable [see Eq. (5)]. In this case the third term in the bracket on the left-hand side of Eq. (15) tends toward zero, and the problem reduces to the familiar boundary element formulation of the exterior problem of solving scattering from a rigid object $\left(\mathbf{A}_{\mathbf{e}}-\mathbf{C}_{\mathbf{e}}\right) \mathbf{p}_{\mathbf{e}}=-4 \pi \mathbf{p}_{\mathrm{e}}^{\mathbf{I}}$. Conversely, if the flow resistivity vanishes (i.e., if the windscreen is removed), $k_{i}=k_{e}$, and therefore $\mathbf{B}_{i}=\mathbf{B}_{e}$ and $\mathbf{A}_{i}=-\mathbf{A}_{e}$ (where the change of sign in the latter expression is due to the 
change of normal vector from the interior to the exterior problem), since the interior and exterior problems involve the same surface. Hence, Eq. (15) reduces to $\left(-\mathbf{C}_{\mathbf{e}}-\mathbf{C}_{\mathbf{i}}\right) \mathbf{p}_{\mathbf{e}}$ $=-4 \pi \mathbf{p}_{\mathbf{e}}^{\mathbf{I}}$ or $\mathbf{p}_{\mathbf{e}}=\mathbf{p}_{\mathrm{e}}^{\mathbf{I}}$, since $\mathbf{C}_{\mathbf{e}}+\mathbf{C}_{\mathbf{i}}=4 \pi \mathbf{I}$ (where $\mathbf{I}$ is the unitary matrix). Thus if the flow resistivity is negligible the windscreen does not change the sound field.

Once Eq. (15) is solved for $\mathbf{p}, \mathbf{u}_{i}$ can be found using Eq. (14), and the pressure inside the windscreen can be found using Eq. (11) with $P$ placed at any location inside the windscreen. If $P$ is strictly inside the windscreen then $C_{i}(P)$ $=4 \pi$.

An intensity probe inside the windscreen could be modeled as described in Ref. 11. However, since windscreens have no influence on sound intensity measurements except at fairly low frequencies ${ }^{4}$ where the probe does not change the sound field at all, ${ }^{11}$ the intensity is simply calculated from the pressure at two points that represent the two microphones, that is, from the expression

$$
\hat{I}_{r}=\frac{\operatorname{Im}\left\{p_{1} p_{2}^{*}\right\}}{2 \omega \rho \Delta r},
$$

where $\Delta r$ is the length of the distance between the microphones.

\section{Infinite windscreen}

If the windscreen is infinitely large its effect on sound intensity measurements can be derived analytically. ${ }^{4}$ The particle velocity follows from Eq. (5)

$$
u_{\mathrm{i} r}=-\frac{1}{j \omega \rho} \frac{\partial p_{i}}{\partial r} \frac{1}{1-j \Phi_{i} / \omega \rho} .
$$

However, the intensity estimate is based on a finite difference approximation to the equation of motion for a lossless medium

$$
\hat{u}_{r}=-\frac{1}{j \omega \rho} \frac{\partial p}{\partial \mathrm{r}},
$$

that is

$$
\hat{u}_{r}=u_{\mathrm{i} r}\left(1-j \Phi_{i} / \omega \rho\right),
$$

from which it follows that the estimated intensity is

$$
\begin{aligned}
\hat{I}_{\mathrm{i} r} & =1 / 2 \operatorname{Re}\left\{p_{i} \hat{u}_{r}^{*}\right\}=\operatorname{Re}\left\{\left(I_{i r}+j J_{i r}\right)\left(1+\frac{j \Phi_{i}}{\omega \rho}\right)\right\} \\
& =I_{i r}-\frac{\Phi_{i}}{\omega \rho} J_{i r}=I_{i r}\left(1-\frac{\Phi_{i}}{\omega \rho} \frac{J_{i r}}{I_{i r}}\right),
\end{aligned}
$$

where $J_{i r}$ is the $r$ component of the reactive sound intensity ${ }^{1,12}$

$$
\mathbf{J}=1 / 2 \operatorname{Im}\{p \mathbf{u} *\} .
$$

The reactive intensity describes the nonpropagating part of the acoustic energy that is merely flowing back and forth. ${ }^{12}$ Many sources have strongly reactive near fields at low frequencies where they essentially generate evanescent waves. ${ }^{1,13}$ In general the reactive intensity points out of a source, corresponding to the radiation impedance being masslike. As can be seen from Eq. (20), the infinite wind-

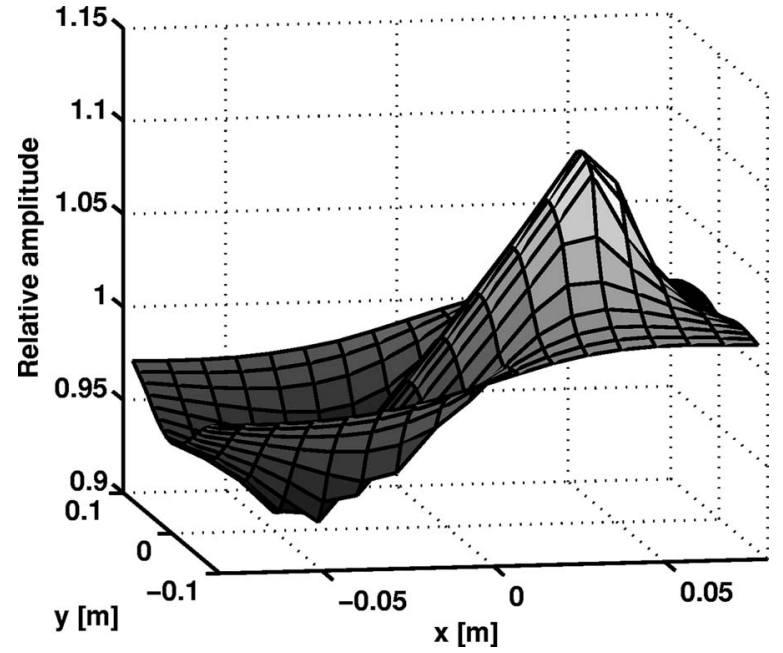

FIG. 1. Effect of spherical windscreen on normalized sound pressure amplitude in the $x-y$ plane. The center of the windscreen is at $(0,0,0)$, and the sound field is generated by a monopole at $(0.10 \mathrm{~m}, 0,0)$.

screen theory predicts a negative bias error that is proportional to the reactive intensity and to the flow resistivity and inversely proportional to the frequency.

\section{NUMERICAL AND EXPERIMENTAL RESULTS}

The coupled BEM model described above has been implemented in MATLAB, and some calculations have been made. Both the spherical and the ellipsoidal windscreen are made of the same material: open-pored polyurethane foam. The flow resistivity of a sample of the material has been measured using the two-microphone method described in Ref. 14 and found to be about $600 \mathrm{kgm}^{-3} \mathrm{~s}^{-1}$.

Figure 1 shows the calculated amplitude of the sound pressure inside and just outside the spherical windscreen when a monopole emitting sound at $63 \mathrm{~Hz}$ is placed at $(x, y, z)=(0.10 \mathrm{~m}, 0,0)$, that is, $10 \mathrm{~cm}$ from the center of the screen, which has a flow resistivity of $600 \mathrm{kgm}^{-3} \mathrm{~s}^{-1}$. The amplitude has been normalized with the distance to the source so as to have a value of unity everywhere in the absence of the windscreen. It is apparent that the pressure amplitude is increased by more than $7 \%$ by the windscreen near the monopole and reduced by a similar fractional amount near the opposite side. However, these modifications should be compared with the much larger pressure variations due to the varying distance to the source.

Figures 2(a) and 2(b) show the calculated phase of the pressure relative to the phase of the volume velocity of the monopole, with and without the spherical windscreen. Close to the monopole the phase angle approximates $90^{\circ}$. As can be seen the windscreen has a significant influence on the phase; in the middle of the screen the sign of the phase gradient is changed. Since the active intensity is proportional to the phase gradient of the pressure ${ }^{12}$ this indicates that the measured sound intensity points in the wrong direction.

Some experiments have also been carried out. A B\&K sound intensity probe of type 3599 with microphones of type B\&K 4181 and the two windscreens UA 0781 and UA 0782 in combination with a B\&K "Pulse" analyzer of type 3560 in 

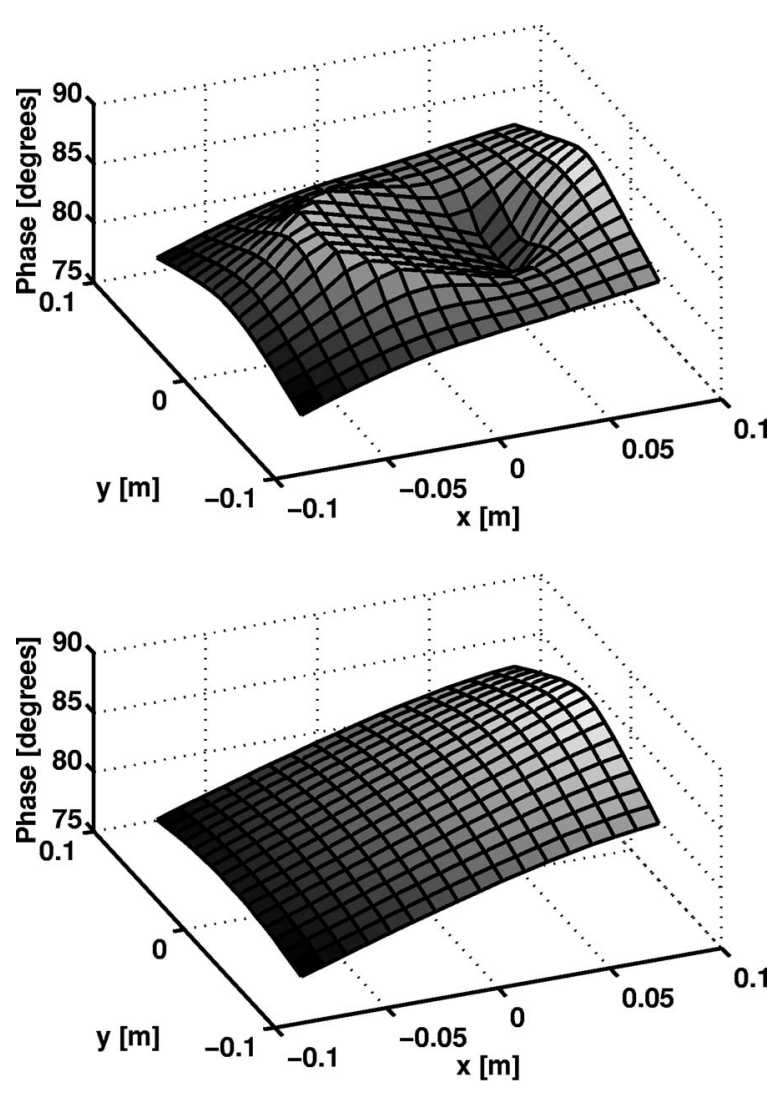

FIG. 2. Phase angle of sound pressure in the $x-y$ plane relative to the volume velocity of the monopole that generates the sound field (a) with and (b) without a spherical windscreen centered at $(0,0,0)$. The monopole is at $(0.10 \mathrm{~m}, 0,0)$.

one-third octave mode were used in the measurements, which took place in DTU's large anechoic room. This room is very good at frequencies down to $50 \mathrm{~Hz}{ }^{15}$ The first measurement was made near a "monopole," a small hole in a large baffle driven by an enclosed loudspeaker on the other side of the baffle; see Fig. 3. Figure 4(a) shows the sound intensity, the reactive intensity, and the particle velocity measured $11 \mathrm{~cm}$ from this source using a 12-mm spacer between the two microphones, without a windscreen on the probe and with the two windscreens. The active and reactive intensities have been normalized by $p_{\mathrm{rms}}^{2} / \rho c$ (where $p_{\mathrm{rms}}$ is the rms pressure without a windscreen on the probe), and the particle

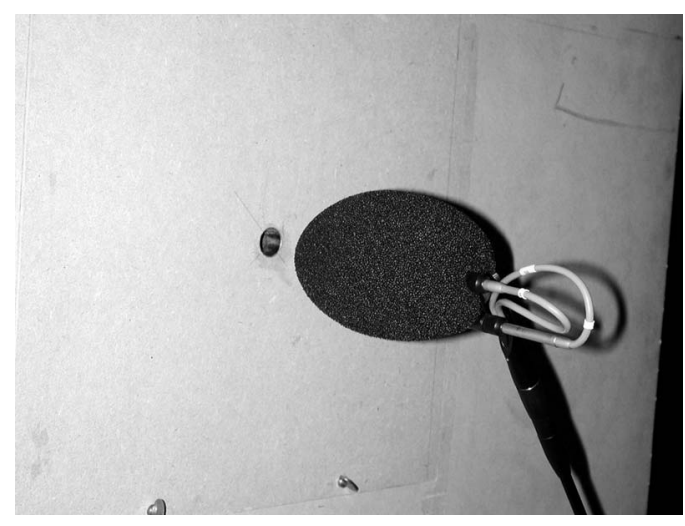

FIG. 3. Measurement near a baffled "monopole" with the ellipsoidal windscreen on the intensity probe.
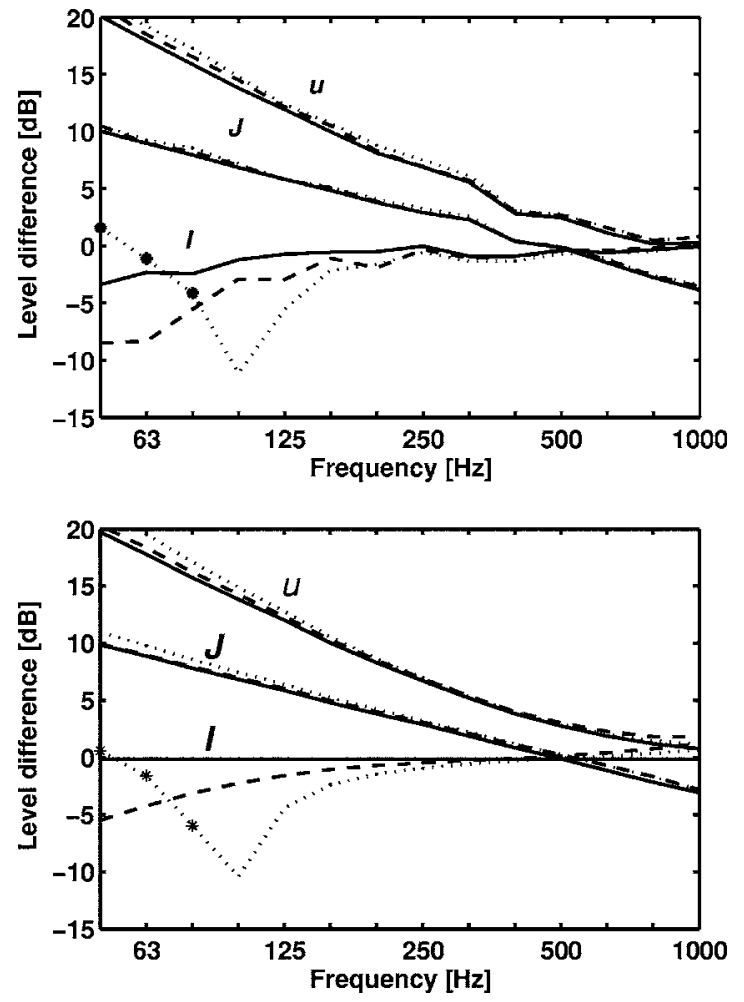

FIG. 4. (a) Measurements and (b) BEM calculations without windscreen (solid line), with an ellipsoidal windscreen (dashed line), and with a spherical windscreen on the intensity probe (dotted line) at a position $11 \mathrm{~cm}$ from a monopole. The stars indicate negative intensity.

velocity has been normalized by $p_{\text {rms }} / \rho c$. Neither windscreen has any significant influence on the measured sound pressure (not shown) or on the particle velocity and reactive intensity. On the other hand the ellipsoidal screen has a considerable influence on the measured active intensity at low frequencies, and the spherical screen has a dramatic influence: It changes the sign of the intensity below $100 \mathrm{~Hz}$. Figure 4(b) shows the results of BEM calculations for the same configurations with $\Phi_{i}=600 \mathrm{kgm}^{-3} \mathrm{~s}^{-1}$ and $\Delta r=12 \mathrm{~mm}$. Considering the difficulty of constructing a "real monopole" there is very good agreement between measurements and calculations. Similar measured and calculated results (not shown) have been obtained with $\Delta r=50 \mathrm{~mm}$ at a distance of $13 \mathrm{~cm}$ from the source, with and without the ellipsoidal windscreen (the spherical windscreen cannot accommodate a 50-mm spacer). No influence of the spacer length was observed.

Another measurement has been carried close to a "dipole" constructed by mounting two loudspeaker units against each other. The moving part of this source, which is shown in Fig. 5, resembles a spherical source of first order (an oscillating sphere), and the reactivity (the ratio of the reactive to the active intensity) at a position on the axis of an oscillating sphere is ${ }^{6}$

$$
\frac{J_{r}(r)}{I_{r}(r)}=\frac{\operatorname{Im}\left\{p(r) u_{r}^{*}(r)\right\}}{\operatorname{Re}\left\{p(r) u_{r}^{*}(r)\right\}}=\frac{1}{k r}+\frac{2}{(k r)^{3}} .
$$

Figure 6(a) shows the measured reactivity at a distance of $21 \mathrm{~cm}$ from the loudspeaker dipole compared with the reactivity the same distances from a spherical source of first order. There is fairly good agreement (except perhaps at 


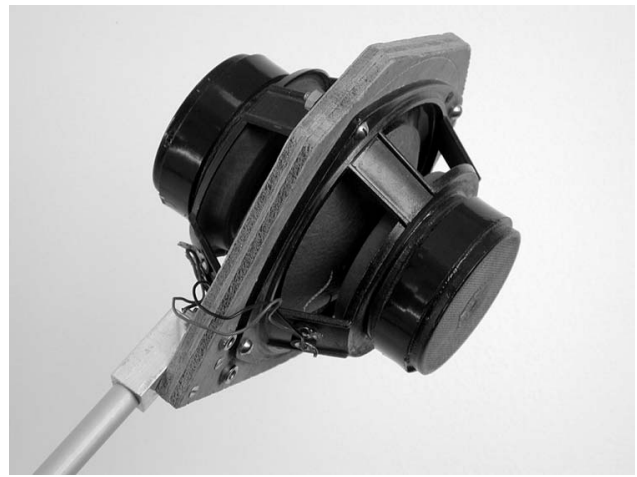

FIG. 5. The loudspeaker dipole.

$80 \mathrm{~Hz}$ ), confirming that the loudspeaker dipole can be modeled reasonably well as such a source (although less well than the experimental monopole described above can be modeled as a real monopole). Figure 6(b) shows the intensity measured with the spherical windscreen on the probe, normalized with the intensity measured without windscreen; the results of BEM calculations with the center of a windscreened intensity probe $21 \mathrm{~cm}$ from the center of a spherical source of first order using a value of $600 \mathrm{kgm}^{-3} \mathrm{~s}^{-1}$ for the flow resistivity; and predictions based on the infinite windscreen theory [Eq. (20)] using the measured reactivity and a value of the flow resistivity found by adjusting it so as to get the best fit. Figure 6(c) shows similar measured and calculated results for the ellipsoidal windscreen. Note (again) that the spherical windscreen has a more serious influence on measurement of sound intensity than the larger ellipsoidal screen; the two windscreens change the sign of the measured intensity below 160 and $125 \mathrm{~Hz}$, respectively. At $50 \mathrm{~Hz}$ the measured intensity with the spherical windscreen on the probe is no less than 50 times $(17 \mathrm{~dB})$ larger than the "true" intensity and pointing in the opposite direction. The influence of the two windscreens is predicted reasonably well by the BEM model considering the fact that the loudspeaker dipole is not a spherical source of first order. The agreement between the measured results and the predictions based on the infinite windscreen theory, Eq. (20), is also fairly good, in particular with the ellipsoidal windscreen (except at $100 \mathrm{~Hz}$ ). However, rather different values of the parameter $\Phi_{i}$ were used in the two cases: $30 \mathrm{kgm}^{-3} \mathrm{~s}^{-1}$ for the ellipsoidal windscreen and $80 \mathrm{kgm}^{-3} \mathrm{~s}^{-1}$ for the spherical windscreen-although they are made of the same porous material and although the actual flow resistivity of the foam is much higher, about $600 \mathrm{kgm}^{-3} \mathrm{~s}^{-1}$. In short, the parameter $\Phi_{i}$ in Eq. (20) is not the actual flow resistivity of the material but a quantity that also depends on the shape and size of the windscreen.

Finally Fig. 7 shows the results of a measurement with the ellipsoidal windscreen on an intensity probe in a standing wave tube with a standing wave ratio of $24 \mathrm{~dB}$. The tube has been constructed for testing sound intensity measurement systems as specified in the IEC and ANSI standards. ${ }^{16,17}$ The tube is $6.2 \mathrm{~m}$ long and has an inner diameter of $29 \mathrm{~cm}$; see Ref. 18 for further details. The results shown in Fig. 7(a) have been obtained at $250 \mathrm{~Hz}$ with an intensity probe cor-
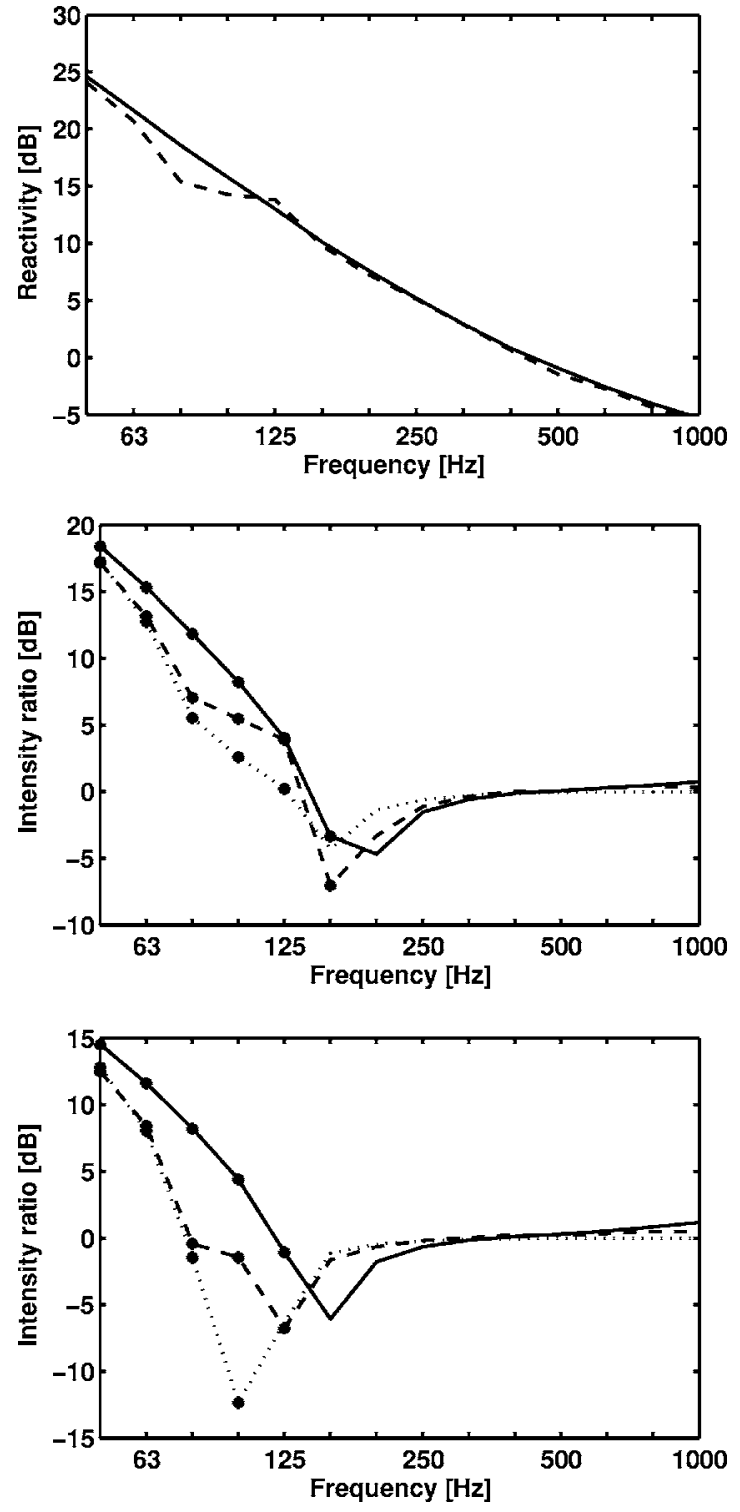

FIG. 6. (a) Reactivity, measured at a distance of $21 \mathrm{~cm}$ (dashed line) from the center of the loudspeaker dipole, and calculated $21 \mathrm{~cm}$ (solid line) from the center of a spherical source of first order; ratio of estimated to "true" intensity with (b) a spherical windscreen and (c) an ellipsoidal windscreen on the probe; measurements (dashed line), BEM calculations (solid line), and infinite windscreen theory (dotted line). The stars indicate negative intensity.

rected for $p$ - $p$ phase mismatch and equipped with a $50 \mathrm{~mm}$ spacer. ${ }^{18}$ As expected the largest deviations from the "true," constant intensity level occur midway between sound pressure maxima and minima where the reactive intensity assumes its largest values. ${ }^{19}$ A prediction calculated with the coupled BEM model is shown in Fig. 7(b), where the amplitude of the incoming wave has been adjusted to match the level in the experiment. In the experiment the sound field at the probe was changed by changing its position in the duct, whereas the same effect has been obtained in the BEM calculations by changing the phase of the reflecting material at one end of the duct. There is very good agreement between the measurements and the BEM calculations. 

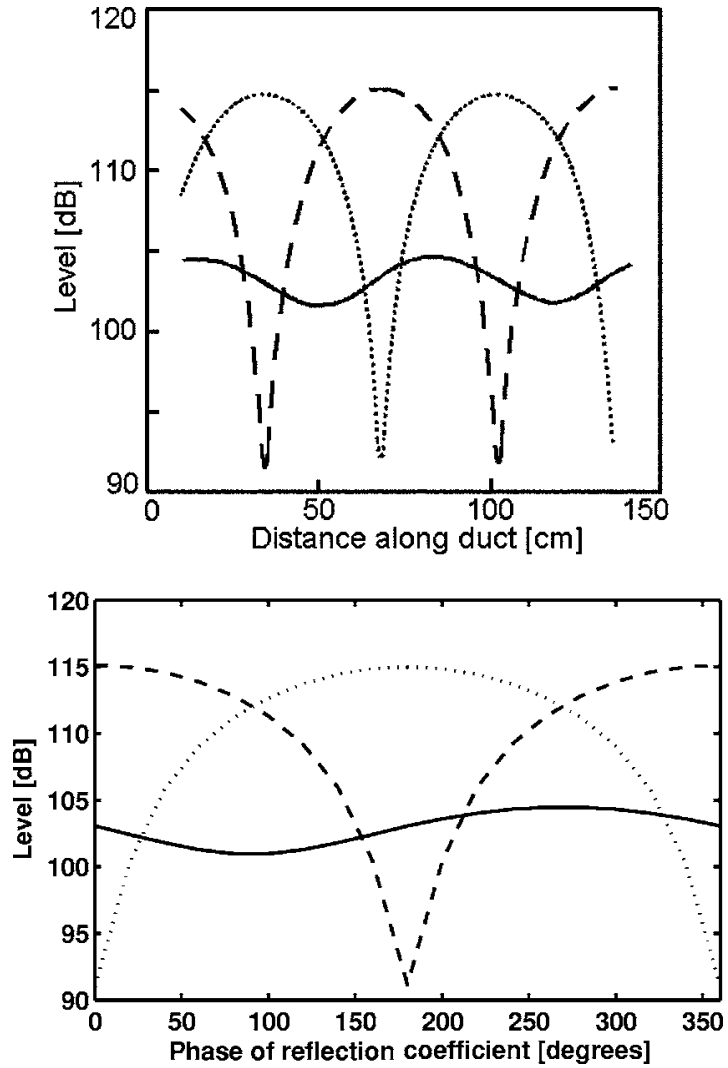

FIG. 7. Measured (a) and predicted (b) levels of sound pressure (dashed line), and particle velocity (dotted line) and intensity (solid line) in a standing wave with a standing wave ratio of $24 \mathrm{~dB}$. Ellipsoidal windscreen with a flow resistivity of $600 \mathrm{kgm}^{-3} \mathrm{~s}^{-1}$.

\section{CONCLUSIONS}

The effect of windscreens of porous foam on sound intensity measurements with the conventional two-microphone method has been examined numerically using a coupled BEM model. The results, which have been validated experimentally, confirm that windscreens give rise to significant bias errors, but only at low frequencies and in strongly reactive sound fields. Under such circumstances the error can be much larger than the intensity itself. By comparison windscreens have almost no effect on measurements of the sound pressure, the particle velocity, and the reactive intensity. The length of the spacer between the microphones does not matter. The size and shape of the windscreen are shown to be important: A large ellipsoidal windscreen gives much smaller errors than a smaller spherical one.

\section{ACKNOWLEDGMENTS}

The authors would like to thank Erling Frederiksen for data obtained with a windscreened sound intensity probe in Brüel and Kjær's standing wave tube for IEC 61043 tests.

${ }^{1}$ F. J. Fahy, Sound Intensity, 2nd ed. (E \& FN Spon, London, 1995).

${ }^{2}$ S. Gade, K. B. Ginn, and R. Hemelrijk, "Sound intensity measurements at 100 km/hour," Proc. Inter-Noise 85, Munich, Germany (Noise Control Foundation, Poughkeepsie, NY, USA, 1985), pp. 1151-1154.

${ }^{3} \mathrm{~F}$. Jacobsen, "Intensity measurements in the presence of moderate airflow," Proc. Inter-Noise 94, Yokohama, Japan (Noise Control Foundation, Poughkeepsie, NY, USA 1994), pp. 1737-1742.

${ }^{4} \mathrm{~F}$. Jacobsen, "A note on measurement of sound intensity with windscreened probes," Appl. Acoust. 42, 41-53 (1994).

${ }^{5}$ C. Zwikker and C. W. Kosten, Sound Absorbing Materials (Elsevier Publishing Company, New York, 1949), see Chap. I.

${ }^{6} \mathrm{P}$. M. Morse and K. U. Ingard, Theoretical Acoustics (McGraw-Hill, New York, 1968), see Sect. 6.2.

${ }^{7}$ J.-F. Allard and Y. Champoux, "New empirical equations for sound propagation in rigid frame fibrous materials," J. Acoust. Soc. Am. 91, 33463353 (1992).

${ }^{8}$ D. K. Wilson, V. E. Ostashev, and S. L. Collier, "Time-domain equations for sound propagation in rigid-frame porous media," J. Acoust. Soc. Am. 116, 1889-1892 (2004).

${ }^{9}$ A. F. Seybert, B. Soenarko, F. J. Rizzo, and D. J. Shippy, “An advanced computational method for radiation and scattering of acoustic waves in three dimensions," J. Acoust. Soc. Am. 77, 362-368 (1985).

${ }^{10} \mathrm{P}$. Juhl, "A numerical study of the coefficient matrix of the boundary element method near characteristic frequencies," J. Sound Vib. 175, 39-50 (1993).

${ }^{11}$ F. Jacobsen, V. Cutanda, and P. M. Juhl, "A numerical and experimental investigation of the performance of sound intensity probes at high frequencies," J. Acoust. Soc. Am. 103, 953-961 (1998).

${ }^{12}$ J. A. Mann III, J. Tichy, and A. J. Romano, "Instantaneous and timeaveraged energy transfer in acoustic fields," J. Acoust. Soc. Am. 82, 17-30 (1987).

${ }^{13}$ E. G. Williams, Fourier Acoustics (Academic Press, San Diego, 1999).

${ }^{14} \mathrm{M}$. Ren and F. Jacobsen, "A method of measuring the dynamic flow resistance and reactance of porous materials," Appl. Acoust. 39, 265-276 (1993).

${ }^{15}$ F. Ingerslev, O. J. Pedersen, P. K. Møller, and J. Kristensen, "New rooms for acoustic measurements at the Danish Technical University," Acustica 19, 185-199 (1967).

${ }^{16}$ IEC 61043 "Electroacoustics-Instruments for the measurement of sound intensity-Measurements with pairs of pressure sensing microphones" (International Electrotechnical Commission, Geneva, Switzerland, 1993).

${ }^{17}$ ANSI S1.9-1996 "Instruments for the Measurement of Sound Intensity" (American National Standards Institute, NY, USA, 1996).

${ }^{18}$ E. Frederiksen, "BCR-report: Free-field intensity sensitivity calibration and standing wave testing," (Brüel and Kjær, Nærum, Denmark, 1992).

${ }^{19}$ F. Jacobsen and E. S. Olsen, "Testing sound intensity probes in interference fields," Acustica 80, 115-126 (1994). 